\title{
Growth and Growth Factors
}

\author{
Régis Coutant \\ Pediatric Endocrinology, University Hospital, Angers, France
}

\begin{abstract}
SOCS2 inactivation, which leads to growth hormone hypersensitivity and overgrowth, is selected as the mechanism of the year in this chapter. The second paper shows that PPAR $\gamma$ plays a role in the growth of male mice. Under new paradigms, we learn that the disruption of a paternally expressed gene from the Gnas locus, XL $\alpha$ s, is associated with poor postnatal growth, and we also discover a new molecular mechanism for Beckwith-Wiedemann syndrome. The effect of growth hormone treatment on the intelligence of subjects born small for gestational age is described in the new hopes section, whereas the associations between fetal growth and later suicidal behavior or breast cancer risk are reported in the new concerns section. Concept revised is represented by one paper showing that height does not influence quality of life in adult women with Turner syndrome previously treated by GH. Under clinical trials, we learn from two studies that early treatment with GH may be beneficial to body composition and motor function of infants with Prader-Willi. Two other papers in this section report the final height of $\mathrm{GH}$-treated children with idiopathic short stature. Under new genes, we discover that TGF $\beta$ receptor-2 mutation is a cause of Marfan syndrome. We then learn that the testicular orphan nuclear receptor TR4, for which a ligand remains to be found, plays an important role in growth. Finally, in the food for thought section GH signaling is further described in 2 papers, one demonstrating a mutant biologically inactive $\mathrm{GH}$, and the other dissecting SOCS2 action on the GH-receptor (GHR) pathway.
\end{abstract}

\section{Mechanism of the year: the growth hormone hypersensitivity syndrome}

\section{SOCS2 negatively regulates growth hormone action in vitro and in vivo}

Greenhalgh CJ, Rico-Bautista E, Lorentzon M, Thaus AL, Morgan PO, Willson TA, Zervoudakis P, Metcalf D, Street I, Nicola NA, Nash AD, Fabri LJ, Norstedt G, Ohlsson C, Flores-Morales A, Alexander WS, Hilton DJ Cancer and Haematology Division, Walter and Eliza Hall Institute of Medical Research, and

Cooperative Centre for Cellular Growth Factors, Parkville, Victoria, Australia greenlaugh@wehi.edu.au

J Clin Invest 2005;115:397-406

Background: The growth hormone (GH)/IGF-1-signaling system is involved in numerous physiological processes during normal growth. SOCS2, a member of the SOCS family, has been showed to be a negative regulator of GH signaling: mice deficient in SOCS2 display an excessive growth phenotype characterized by a $30-50 \%$ increase in mature body size. The present study explores the mechanisms by which SOCS2 regulates GH signaling.

Methods: GH administration to mice lacking SOCS2 and endogenous GH (SOCS2 ${ }^{-1-}$ Ghrhrlit/lit) and characterization of the interaction between SOCS2 and the GH receptor using structure/function and biochemical interaction studies.

Results: The SOCS2 $2^{-1-}$ overgrowth phenotype was abolished in mice deficient in endogenous GH. However, treatment with exogenous $\mathrm{GH}$ induced excessive growth in mice lacking both endogenous GH and SOCS2. Microarray analysis on liver RNA extracts from male SOCS2 ${ }^{-1-}$ Ghrhrlit/lit compared to SOCS2 ${ }^{+/+}$Ghrhrlit/lit mice injected with GH showed that SOCS2 $2^{-/-}$Ghrhrlit/lit mice demonstrated a hyperresponsiveness to $\mathrm{GH}$, which was evident for well-known GH-regulated genes, such as IGF-1. Analysis of the 3 structural motifs of the SOCS2 molecule, the N-terminal domain, the central SH2 domain, and the C-terminal domain termed the SOCS box, revealed that each plays a crucial role in SOCS2 function, with the conserved SOCS-box motif being essential for all inhibitory function. SOCS2 was found to bind 2 phosphorylated tyrosines on the GH receptor, and mutational analysis of these amino acids showed that both were essential for SOCS2 function.

Conclusion: The data provide evidence that SOCS2 is a negative regulator of GH signaling. 
SOCS2 knockout mice exhibit gigantism characterized by an increase in body weight and length, and thickening of dermal layers [1]. There were no detectable hematological abnormalities, although SOCS is involved in cytokine signaling. The phenotype was very similar to that observed in GH excess syndrome. Overgrowth in the SOCS2 ${ }^{-l-}$ mice was not apparent from birth and only seen after postnatal week 3. IGF-1 mRNA was elevated in some tissues, but circulating IGF-1 was not increased in these mice, indicating that liver IGF-1 production was normal. In this paper, the authors observed that the overgrowth phenotype was abolished when SOCS2 ${ }^{-1-}$ mice were crossed with mice deficient in $\mathrm{GH}$, thus indicating that it was dependent on $\mathrm{GH}$. They further demonstrated interactions of SOCS2 with the GH receptor. Finally, they showed upregulation of $\mathrm{GH}$-dependent genes (including IGF-1) upon GH treatment in SOCS2 ${ }^{-1-}$ mice with GH deficiency. Overall, they described a very convincing ' $\mathrm{GH}$ hypersensitivity syndrome'. It is tempting to speculate that the human disease, secondary to SOCS2-inactivating mutations, could be described in the future: the predicted phenotype from the murine model would be gigantism with normal GH secretion and normal or high IGF-1 levels, which may be frequent in tall stature. Furthermore, SOCS2 may be a promising target for treating disorders of the GH/IGF-1 system.

This paper is also important for the $\alpha$-screen assay technology used, which forms a basis of a highthroughput method to screen chemical compound libraries for small-molecule inhibitors of SOCS2$\mathrm{GH}$ receptor interactions. Verification of SOCS2-GH receptor peptide interactions was performed using an AlphaScreen (PerkinElmer Life Sciences) interaction assay. The $\alpha$-screen assay utilizes donor beads coated with phosphorylated peptide and acceptor beads coated with recombinant SOCS2-SH2 domain protein. Interaction between phosphopeptide and SOCS2 brings the acceptor and donor beads into close proximity, and excitation of the donor beads by laser light induces production of highly reactive singlet oxygen that diffuses from the donor bead before rapidly decaying. When an acceptor bead is held in close proximity to the donor bead, singlet oxygen reacts with a reagent in the acceptor beads to generate chemiluminescence. The intensity of the fluorescence output therefore provides a quantifiable measurement of the SOCS2/phosphopeptide-binding interaction.

\section{New mechanisms}

A link between fat metabolism and growth hormone signaling

\section{Altered growth in male peroxisome proliferator-activated receptor gamma (PPAR $\gamma$ ) heterozygous mice: involvement of PPAR $\gamma$ in a negative feedback regulation of growth hormone action}

Rieusset J, Seydoux J, Anghel SI, Escher P, Michalik L, Soon Tan N, Metzger D, Chambon P, Wahli W, Desvergne B

Center for Integrative Genomics, NCCR Frontiers in Genetics, University of Lausanne, Switzerland

Mol Endocrinol 2004; 18:2363-2377

Background: The peroxisome proliferator-activated receptor- $\gamma(\operatorname{PPAR} \gamma)$ plays a major role in fat tissue development and physiology. The authors investigated the growth characteristics of the PPAR $\gamma^{+1-}$ mice. Methods: Study of growth and the somatotropic axis of PPAR $\gamma^{+/-}$mice under standard conditions or with treatment with MSG, an agent inducing hypothalamic lesion, obesity, and pituitary insufficiency. Results: Female PPAR $\gamma^{+-}$mice had normal growth, whereas male PPAR $\gamma^{+1-}$ mice showed a 5-6\% decrease in weight and length compared to wild-type males from the weaning period up to 24 weeks, with both lean and fat mass affected. The growth alteration of the male PPAR $\gamma^{+/-}$mice was aggravated by MSG treatment. Treatment of male wild-type mice with a PPAR $\gamma$ antagonist induced a similar growth-retarded phenotype to that observed in male PPAR $\gamma^{+/-}$mice. The levels of circulating GH and that of its downstream effector, IGF-I, are not altered in mutant mice. However, the IGF-I mRNA level is decreased in the white adipose tissue (WAT) of PPAR $\gamma^{+/-}$mice and is not changed by acute administration of recombinant human $\mathrm{GH}$, suggesting an altered $\mathrm{GH}$ action in the mutant animals. Importantly, expression of the gene encoding the suppressor of cytokine signaling-2, which is an essential negative regulator of GH signaling, is strongly increased in the WAT of PPAR $\gamma^{+/-}$mice.

Conclusion: Although the relationship between the altered GH signaling in the WAT and reduced body size remains unclear, these results suggest a novel role of PPAR $\gamma$ in GH signaling. 
The peroxisome proliferator-activated receptors are ligand-activated transcription factors that belong to the nuclear hormone receptor superfamily. They are known to function as heterodimers with the retinoid $X$ receptor. PPARs have been implicated in diverse biological pathways such as lipid and glucose homeostasis, control of cell proliferation and differentiation. PPAR $\gamma$ plays a key role in adipocyte differentiation as well as in the maintenance of differentiated mature adipocytes. Its natural ligands comprise polyunsaturated fatty acids and some of their metabolites. The insulin sensitizers thiazolidinediones (TZDs) are synthetic high-affinity ligands of PPAR $\gamma$. This study showed the involvement of PPAR $\gamma$ in postnatal growth and pointed to a complex regulatory network that involves the GH/IGF-I axis and its modulation via SOCS-2 expression. Interestingly, the paper shows the sexual dimorphism of the PPAR $\gamma^{+1-}$ phenotype that includes both the body size (herein) and the susceptibility to develop insulin resistance upon a high-fat diet. Females express lower levels of SOCS- 2 and IGF-I mRNA than males. PPAR $\gamma^{+l-}$ females and males present similar alterations of the expression of these genes. However, the difference in expression levels of SOCS-2 and IGF-I between males and females is likely to contribute to the metabolic sexual dimorphism, particularly with respect to insulin sensitivity.

\section{New paradigms}

Paternal deficiency of XL $\alpha$ s: a human disease yet to be discovered

\section{The imprinted signaling protein $X L \alpha s$ is required for postnatal adaptation to feeding}

Plagge A, Gordon E, Dean W, Boiani R, Cinti S, Peters J, Kelsey G

Laboratory of Developmental Genetics and Imprinting, Babraham Institute, Cambridge, UK

Nat Genet 2004;36:818-826

Background: Imprinting is an epigenetic phenomenon characterized by parental allele-specific differences in gene expression. Genomic imprinting and the genes concerned in the control of fetal growth are now better known. The imprinting of Gnas, the gene encoding the stimulatory G-protein subunit, is one of the most studied. The Gnas complex encodes the G protein- $\alpha$-stimulating peptide (Gs $\alpha$ ), which interacts with adenylate cyclase and numerous related transcripts.

Methods: The authors disrupted a paternally expressed transcript at the Gnas locus, Gnasxl, that encodes a protein, XL $\alpha$ s, in which the N-terminal end of Gs is replaced by a large acidic XL domain. XL $\alpha$ s retains all the functional domains and many biochemical properties of Gs $\alpha$, including binding to subunits and activation of adenylate cyclase.

Results: Mice with mutations in Gnasxl have low birth weight, poor suckling, neonatal hypoglycemia, low levels of circulating insulin and glucagon, reduced brown fat, and poor postnatal growth and survival. Increased cAMP levels were found in brown adipose tissue of Gnasxl mutants. In addition, Gnasxl was found to be expressed at early postnatal stages in the hypothalamus, the pituitary, the adrenal medulla, the adipose tissue, and the pancreas, which is consistent with a its putative role in energy homeostasis.

Conclusion: These findings suggest that XL $\alpha$ s can antagonize Gs $\alpha$-dependent signaling pathways. The opposing effects of maternally and paternally expressed products of the Gnas locus provide tangible molecular support for the parental-conflict hypothesis of imprinting (fig. 1).

Last year, we learned that the renal phenotype of pseudohypoparathyroidism is imprinted from the father. In this study, the authors report the phenotype associated with disruption of the paternally expressed XL $\alpha$ s gene in mice: low birth weight, poor suckling, reduced brown fat, and poor postnatal growth and survival. This phenotype probably results from the overuse of existing energy reserves and an inability to replenish lipid stores. The $X L \alpha s$ gene encodes an extra-large variant of the stimulatory $G$ protein $\alpha$ subunit which can bind to $\beta \gamma$ subunits and activate adenylate cyclase. As both Gnas and Gnasxl are expressed in adipose tissues, the increase in CAMP in brown adipose tissue from these mice suggests that XLas plays an inhibitory or interfering role in CAMP signaling of brown adipocytes. The observed phenotype was in accordance with the parental conflict hypothesis 


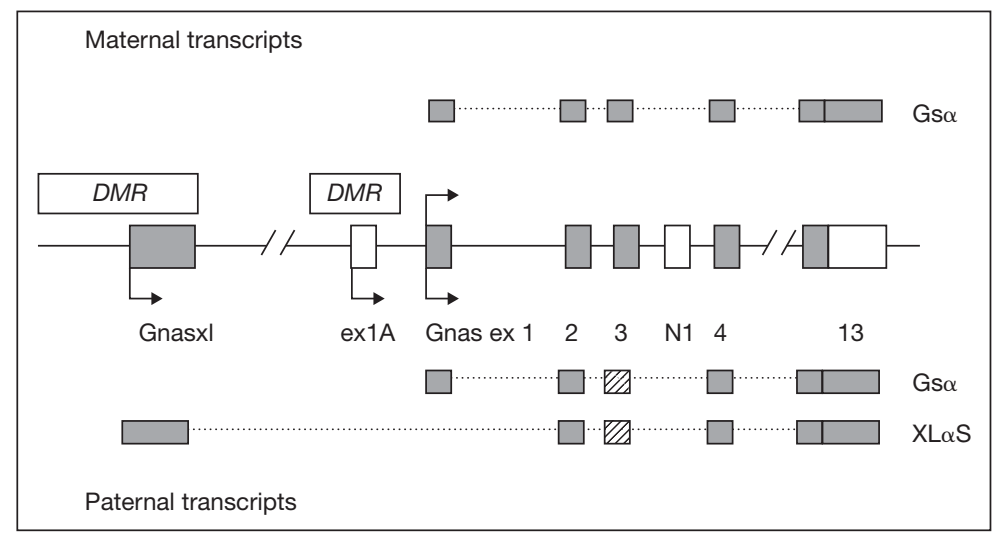

Fig. 1. Open boxes represent noncoding sequences, solid boxes represent coding sequences, and hatched boxes represent exons that are alternatively skipped; exons 5-12 are omitted. DMR (differentially methylated region) in italics indicates DMRs methylated on the maternal allele.

of imprinting, which implies that genomic imprinting allows the paternal genome to promote growth of the fetus and the maternal genome to inhibit growth. It further extends the parental conflict hypothesis to the postnatal period. The consequences of disruption of the paternal XL $\alpha$ s gene are not known in humans: the present study suggests that there may be other human disease phenotypes waiting to be discovered. One would predict a subject with low birth weight and slow postnatal growth, hypotonia, hypoglycemia, and a defect in adipose tissue storage.

\section{IGF-2: from overgrowth to growth restriction}

\section{Microdeletions in the human H19 DMR result in loss of IGF2 imprinting and Beckwith-Wiedemann syndrome}

Sparago A, Cerrato F, Vernucci M, Ferrero GB, Silengo MC, Riccio A

Dipartimento di Scienze Ambientali, Seconda Università di Napoli, Caserta, Italy

Nat Genet 2004;36:958-960

Background: The overgrowth- and tumor-associated Beckwith-Wiedemann syndrome (BWS) results from dysregulation of imprinted genes on chromosome 11p15.5. Upregulation of IGF2 is important for the somatic overgrowth and tumor predisposition of BWS. A subclass of individuals with BWS shows hypermethylation and silencing of the maternal $\mathrm{H} 19$ allele and activation of both parental IGF2 alleles. In mice, imprinting of the paternally expressed IGF2 and maternally expressed H19 genes is controlled by a 2-kb differentially methylated region (DMR) located 5' of H19.

Methods: The authors analyzed 7 individuals with BWS with $H 19$ hypermethylation for the presence of deletions in the H19 DMR.

Results: Inherited microdeletions in the H19 DMR were found in 2 individuals. Maternal transmission of the deletions results in hypermethylation of the H19 DMR, biallelic IGF2 expression, H19 silencing and BWS, indicative of loss of function of the IGF2-H19 imprinting control element.

Conclusion: This indicates that at least some of the DNA-methylation defects are caused by mutation in an imprinting regulatory region.

BWS is a clinically heterogeneous disorder which may include overgrowth, hemihypertrophy, macroglossia, abdominal wall defects, embryonal tumors, visceromegaly, and neonatal hypoglycemia. Most cases of BWS occur sporadically and are believed to result from 'pure' epigenetic errors on chromosome $11 \mathrm{p} 15$. The 3 most frequent findings identified are IGF-2 loss of imprinting, observed in cases 
with uniparental paternal disomy, CDKN1C gene loss-of-function, and alteration in the imprinting center KVLQT1 DMR. This report describes a fourth mechanism associated with BWS, microdeletions in the maternal H19 DMR, between the IGF-2 and the H19 genes. H19 encodes for an apparently untranslated RNA transcript. Microdeletions in H19-DMR prevented the binding of CTCF, an insulator protein, thus allowing interaction between IGF-2 promoters and enhancers on the maternal allele, and biallelic IGF-2 expression (instead of expression from the paternal allele only). IGF2 is a central player in epigenetics and BWS, and the overgrowth phenotype is dependent upon the IGF2 mRNA level. Either the paternally expressed IGF-2 gene is duplicated (uniparental paternal disomy), or the normally silenced maternal IGF-2 gene is 'paternalized', leading to its activation. Whether the microdeletions in the H19-DMR are sufficient to cause BWS has been recently discussed [2], since they were not always associated with BWS phenotype. This observation favors a 'threshold model' in which IGF2 expression levels that exceed a threshold are critical in the etiology of BWS and its associated tumors. Interestingly, duplication of the maternal 11 p15 region, which would theoretically lead to a defect in IGF-2 expression, has been found in 2 of 46 patients with Silver-Russell syndrome with a somewhat opposite phenotype to BWS [3].

\section{New hopes \\ GH for refueling the brain}

\section{Intelligence and psychosocial functioning during long-term growth hormone therapy in children born small for gestational age}

Van Pareren YK, Duivenvoorden HJ, Slijper FS, Koot HM, Hokken-Koelega AC

Sophia Children's Hospital/Erasmus MC, Department of Pediatrics, Division of Endocrinology, Rotterdam, the Netherlands

J Clin Endocrinol Metab 2004;89:5295-5302

Background: Subjects born small for gestation age (SGA) may have lowered intelligence, poor academic achievement, and behavioral problems. The authors investigated the effect of GH treatment on intelligence and psychosocial functioning in SGA children.

Methods: Randomized, double-blind, GH-dose response study in SGA children. Patients received 1 or $2 \mathrm{mg} \mathrm{GH} / \mathrm{m}^{2}$ body surface. Intelligence and psychosocial functioning were evaluated at the start of GH treatment, after 2 years of GH treatment, and in 2001. Values are expressed as means \pm SEM.

Results: Birth length SD score was $-3.6 \pm 0.2$, age and height at the start of GH treatment was $7.4 \pm 0.2$ years and $-3.0 \pm 0.1 \mathrm{SD}$ score, respectively. The subjects received GH therapy for $8.0 \pm 0.2$ years, and mean age in 2001 was $16.5 \pm 0.3$ years. Block-design s-score (a performance IQ subtest) and the estimated total IQ significantly increased from scores lower than Dutch peers at the start to comparable scores at the end of the study. The increase over time for the Vocabulary s-score (a verbal IQ subtest) was not significant. Externalizing Behavior SD scores and Total Problem Behavior SD scores improved significantly during GH therapy to scores comparable to Dutch peers. Self-perception SD scores improved from the start of GH treatment until 2001 to scores significantly higher than Dutch peers. No significant differences between the $2 \mathrm{GH}$ dosage groups were found. Improvement in Externalizing and Total Problem Behavior SD scores over time was significantly related to a change in height SD score.

Conclusion: IQ, behavior and self-perception showed a significant improvement over time from scores below average to scores comparable to Dutch peers.

Impaired fetal growth, possibly caused by inadequate fetal nutrition, may affect mental development, which was shown to be dependent on the severity of the growth restriction and the absence of catch-up growth [4]. This is one of the first studies to longitudinally examine the intellectual, social-emotional, and behavioral functioning of children born SGA. It showed that GH treatment in subjects born SGA is associated with improved performance in nonverbal intelligence, whereas verbal intelligence remained within the lower limits of the average range. Whether the increase in test scores corresponded to a direct effect of $\mathrm{GH}$ itself on cerebral functioning is unknown. It should be remembered, however, that this is a longitudinal study, without a control group and that the 
untreated outcome in a similar group of patients was not assessed. Benefits of GH treatment have been previously shown on cognition, energy, mood, and behavior in $\mathrm{GH}$-deficient children and adults [5]. However, the extra medical attention associated with $\mathrm{GH}$ treatment might be an alternative explanation for the improvement of IQ and psychosocial functioning. Whether the improvement in IQ, behavior, and self-perception effectively translates into better academic achievement would be the next important step to study. Nevertheless, this, as well as other works published this year (see below clinical trials), suggests that GH treatment, beyond the height gain, may improve 'brain performance'. Will this become a new therapeutic aim for GH?

\section{New concerns \\ Suicidal behavior: early growth matters}

\section{Restricted fetal growth and adverse maternal psychosocial and socioeconomic conditions as risk factors for suicidal behavior of offspring: a cohort study}

Mittendorfer-Rutz E, Rasmussen F, Wasserman D

Swedish National and Stockholm County Council Centre for Suicide Research and

Prevention of Mental III-Health (NASP), National Institute of Psychosocial Medicine, Stockholm, Sweden

Lancet 2004;364:1135-1140

Background: The goal of this study was to assess the effects of early fetal environment and parental factors on suicidal behavior in adolescents and young adults.

Methods: Intrauterine and perinatal conditions, and two maternal variables (psychosocial factors as captured by age and parity, and socioeconomic status as reflected by the education level) were registered in a cohort of 713,370 individuals born in Sweden between 1973 and 1980 and followed-up until December 31, 1999. Hazard ratios were calculated.

Results: Multivariate analyses revealed that low birth weight adjusted for gestational age $(<2,500 \mathrm{~g}$; $2.23,95 \%$ CI $1.43-3.46, \mathrm{p}<0.0001$ ) or being born to a mother still in adolescence (age $<19$ years at time of delivery; $2.30,95 \%$ CI 1.64-3.22, $\mathrm{p}<0.0001)$ increased the risk of later suicide. For suicide attempts, low birth length adjusted for gestational age $(<47 \mathrm{~cm} ; 1.29,95 \%$ CI $1.18-1.41, \mathrm{p}<0.0001)$ and having an adolescent mother $(2.09,95 \%$ CI $1.89-2.32, \mathrm{p}<0.0001)$ increased risk, as did low level of maternal education $(1.36,95 \%$ CI $1.27-1.46, \mathrm{p}<0.0001)$ and being 4 th or later in birth order $(1.79,95 \%$ CI 1.62-1.97, $\mathrm{p}<0.0001)$.

Conclusion: Multiparity and low maternal education predicted suicide attempts, whereas restricted fetal growth and teenage motherhood were associated with both suicide completion and attempts in offspring. The effect of these factors on suicide risk was mild and less than the risk conferred by family history of suicidal behavior.

This study showed that intrauterine and perinatal environment might have independent effects on future suicidal risk. This is another association between impaired fetal growth and brain functioning. It further extends the concept of fetal programming of adult diseases. In rodents, cross-adoption studies showed that mice with low anxiety express significantly more anxiety if they are both transplanted into the uteri of anxious dams and reared by anxious dams. Genetically identical low-anxiety mice that are either only reared by an anxious dam or only transplanted to an anxious dam's uterus do not show exacerbation of anxiety behaviors [6]. These experiments, together with the human observations, show that intrauterine and postnatal environment both play crucial roles in programming future behavior. 


\section{Growth patterns and the risk of breast cancer in women}

Ahlgren M, Melbye M, Wohlfahrt J, Sorensen TI

Department of Epidemiology Research, Danish Epidemiology Science Center, Statens Serum Institute,

Copenhagen, Denmark

abk@ssi.dk

N Engl J Med 2004;351:1619-1626

Background: The authors investigated whether fetal growth or growth during childhood and adolescence influence the risk of later breast cancer.

Methods: A cohort of 117,415 Danish women was studied, corresponding to a follow-up of 3,333,359 person-years. Birth weight, age at menarche, and annual measurements of height and weight were obtained from school health records. Vital status, age at first childbirth, parity, and diagnosis of breast cancer were obtained through national registries.

Results: High birth weight (relative risk 1.1 per kg), high stature at 14 years of age (RR 1.1 per $5 \mathrm{~cm}$ ), low body-mass index (RR 0.97 per unit of BMI at 14 years of age), and peak growth at an early age (RR 0.97 per year of age at peak growth) were independent risk factors for breast cancer. Height at 8 years of age and pubertal growth were also associated with breast cancer. The attributable risks of birth weight, height at 14 years of age, BMI at 14 years of age, and age at peak growth were $7,15,15$, and $9 \%$, respectively. Age at menarche, age at first childbirth, and parity had no effect on these risks.

Conclusion: Fetal growth and growth during childhood and adolescence influence the risk of breast cancer.

Previous epidemiological studies have linked tall stature with breast cancer [7]. In this work, the authors further refined this association by studying the relationships between growth pattern and risk of breast cancer. They found that high birth weight, rapid growth around the time of breast development, high stature, and low BMI during adolescence were independent risk factors for breast cancer later in life. Age at menarche was not related to the risk of breast cancer. In Japan, where a large increase in height has been observed over the past five decades, presumably primarily because of a change in diet, the increase in height has been followed by a steady increase in the rate of breast cancer [8]. Although causality cannot be inferred from these associations, it is tempting to speculate about the genetic or molecular mechanisms linking growth with cancer. For instance, high birth weight and tall stature could be related to genetically determined high levels of IGF1 or other growth factors that affect breast cells later during adulthood. Generally, the world population has become taller over time, especially during the past century. At least part of the increasing incidence of breast cancer may be attributable to this growth. In that respect, we have no evidence to support a relationship between $\mathrm{GH}$ therapy and breast cancer; but have we really studied it in depth? On the other hand, higher birth weight, higher stature, and lower BMI during adolescence are related to lower risks of cardiovascular disease and diabetes: the fetal growth as well as the growth pattern during childhood and adolescence may balance the later risks for cancer or cardiovascular disease. Between cancer and cardiovascular disease, the healthy path may be difficult to find. 


\section{Quality of life determinants in young women with Turner syndrome after growth hormone treatment: results of the StaTur population-based cohort study}

Carel JC, Ecosse E, Bastie-Sigeac I, Cabrol S, Tauber M, Leger J, Nicolino M, Brauner R, Chaussain JL, Coste J

Pediatric Endocrinology and Institut National de la Santé et de la Recherche Médicale Unité 561,

Groupe Hospitalier Cochin-Saint Vincent de Paul, Paris, France

carel@paris5.inserm.fr

J Clin Endocrinol Metab 2005;90:1992-1997

Background: GH treatment in children with Turner syndrome has been used for more than 20 years. The authors studied quality-of-life determinants in a population-based cohort of young women with Turner syndrome who had previously been treated with GH.

Methods: 891 women aged over 18 years and recorded in the French Growth Hormone Register were eligible, 818 were available and 568 participated (69\%). Demographic characteristics, health status, sexual life, treatment expectations, quality-of-life scores from 2 questionnaires (Medical Outcome Study Short Form 36 (SF-36), and General Health Questionnaire 12) were determined.

Results: They were $22.6 \pm 2.6$ years old (mean \pm SD) at time of participation, measured $150.9 \pm$ $5.6 \mathrm{~cm}$, and had received GH for $4.8 \pm 2.2$ years. There was no difference in SF-36 scores between participants and French women of the general population. Height and estimated height gain from treatment were not associated with quality-of-life scores. However, higher expectations from treatment were associated with lower quality of life. Cardiac involvement (detected during childhood or reported, $12 \%$ of participants), otological problems (26\% of participants), or induction of puberty after 15 years of age (34\% of participants) was associated with lower scores for at least one of the SF-36 dimensions.

Conclusion: Quality of life is normal in young adults with Turner syndrome treated with GH and is not dependent on height. General health and otological care should be given appropriate attention in the care of children with Turner syndrome.

From randomized studies [9, 10], and from spontaneous growth curves of girls with Turner syndrome, one can estimate the height gain with the mean $\mathrm{GH}$ dose used in this report to about $5-7 \mathrm{~cm}$. However, the authors showed that patients may be smarter than their endocrinologists: height was not the most important health concern in adult women with Turner syndrome who had been treated with $\mathrm{GH}$ during childhood. The patients who had the highest height gain expectations from the treatment had the lowest quality-of-life (QOL) score, which seemed somewhat contradictory with the absence of importance of height on QOL: this at least suggests that some of the patients perceived a relative 'treatment failure' which may have influenced their scores of quality of life. The extra medical attention associated with $\mathrm{GH}$ treatment, or the intervention itself (GH treatment) rather than its result (height increment) might have improved perceived health-related quality of life: $88 \%$ of the young adults had a favorable opinion of the treatment they had received. Importantly, this study identified 3 conditions, otological disease, cardiac malformation, and late pubertal induction as having a clear effect on QOL score. For most pediatricians, these would rank far behind height! Although Turner syndrome patients clearly benefit from GH therapy, it is now clear that we have to focus on dimensions other than height. In this respect, one important question is the impact of infertility on QOL scores? 


\title{
Growth hormone improves mobility and body composition in infants and toddlers with Prader-Willi syndrome
}

\author{
Carrel AL, Moerchen V, Myers SE, Bekx MT, Whitman BY, Allen DB \\ Department of Pediatrics, University of Wisconsin Children's Hospital, Madison, Wisc., USA \\ alcarrel@wisc.edu
}

J Pediatr 2004;145:744-749

Background: The authors tested the hypothesis that GH treatment of infants and toddlers with PraderWilli syndrome (PWS) could speed accretion of lean body mass, reduce accumulation of fat mass, and improve motor function.

Methods: Twenty-nine subjects with PWS (4-37 months of age) were randomized to GH treatment $\left(1 \mathrm{mg} / \mathrm{m}^{2} /\right.$ day) or observation for 12 months. Body composition and bone mineral density were measured by dual X-ray absorptiometry; energy expenditure was measured by doubly labeled water; and motor constructs of mobility $(\mathrm{M})$ and stability $(\mathrm{S})$ were assessed using the Toddler Infant Motor Evaluation (TIME).

Results: GH-treated subjects had decreased percent body fat (mean $22.6 \pm 8.9$ vs. $28.5 \pm 7.9 \%$; $\mathrm{p}<0.001$ ), increased lean body mass (mean $9.82 \pm 1.9$ vs. $6.3 \pm 1.9 \mathrm{~kg} ; \mathrm{p}<0.001$ ), and increased height velocity $Z$ scores (mean $5.0 \pm 1.8$ vs. $1.4 \pm 1.0 ; \mathrm{p}<0.001$ ) vs. controls. Higher mobility skill acquisition was observed in patients who began GH before 18 months of age by comparison with controls within the same age range (mean increase in raw score $284 \pm 105$ vs. $206 \pm 63 ; \mathrm{p}<0.05$ ).

Conclusion: GH treatment of infants and toddlers with PWS for 12 months significantly improves body composition and when begun before 18 months of age increases mobility skill acquisition.

\section{Growth hormone and body composition in children younger than 2 years with Prader-Willi syndrome}

Eiholzer U, L'Allemand D, Schlumpf M, Rousson V, Gasser T, Fusch C

Department of Biostatistics, University of Zurich, Zurich, Switzerland

mail@childgrowth.org

J Pediatr 2004;144:753-758

Background: The authors tested the hypothesis that GH treatment of infants and toddlers with PWS could improve body composition and prevent massive obesity.

Methods: During 30-month GH therapy $\left(6 \mathrm{mg} / \mathrm{m}^{2}\right.$ per week) 11 children with PWS aged $<2$ years were compared with 6 infants administered only coenzyme Q10 $\left(6 \mathrm{mg} / \mathrm{m}^{2}\right.$ per week). Body composition was measured by deuterium dilution.

Results: Lean mass adjusted for height decreased by $-0.46 \pm 0.3 \mathrm{SD}(\mathrm{p}<0.05)$ per year in the Q10 group but rose by $0.25 \pm 0.3 \mathrm{SD}(\mathrm{p}<0.05)$ per year during $\mathrm{GH}$ therapy, normalizing after 30 months $(-0.70 \pm 1.0 \mathrm{SD})$. Percent fat mass for age was above the normal average (GH group, $31.0 \pm 4.5 \%$, Q10 group, $32.4 \pm 9.5 \%$ ), increased by $0.71 \pm 0.7$ SD per year in the Q10 group, and remained stable up to 30 months in the GH-treated group.

Conclusion: Early institution of GH may restore lean body mass and delay fat tissue accumulation.

PWS is increasingly diagnosed in infancy. GH therapy instituted during childhood improves but does not normalize body composition, energy expenditure, and strength and agility in PWS. These reports show that early treatment with GH significantly improves body composition of children with PWS, leading to normalization of lean mass, not fat mass, after 30 months of treatment. In addition, mobility skills were found to be increased in the younger subjects, with no improvement in the older ones. This underlines another potential effect of GH treatment, beyond height and body composition. Whether this improvement, as measured by specific scales, effectively translates into clinical benefit awaits further confirmation. This year, further reports on sudden death in PWS during $\mathrm{GH}$ therapy were published and a special warning was added to the $\mathrm{GH}$ indication for this condition 
$[11,12]$. This justifies further epidemiological studies to determine more accurately whether this association is causal, and better understand its pathogenesis.

\section{GH for every short child?}

\section{Effect of growth hormone treatment on adult height in peripubertal children with idiopathic short stature: a randomized, double-blind, placebo-controlled trial}

Leschek EW, Rose SR, Yanovski JA, Troendle JF, Quigley CA, Chipman JJ, Crowe BJ, Ross JL, Cassorla FG, Blum WF, Cutler GB Jr, Baron J

Developmental Endocrinology Branch, National Institute of Child Health and Human Development/NIH, Bethesda, Md., USA

J Clin Endocrinol Metab 2004;89:3140-3148

Background: Whether GH treatment increases adult height in subjects with idiopathic short stature is not known.

Methods: Randomized, double-blind, placebo-controlled trial to determine the effect of GH on adult height in peripubertal children. Subjects ( $\mathrm{n}=68 ; 53$ males and 15 females), 9-16 years old, with ISS (height or predicted height $<$ or $=-2.5$ SDS) received either GH $(0.074 \mathrm{mg} / \mathrm{kg})$ or placebo s.c. three times per week until they were near adult height.

Results: Adult height after a mean treatment duration of 4.4 years was greater in the GH-treated group $(-1.81 \pm 0.11$ SDS, available in 22 subjects) than in the placebo-treated group $(-2.32 \pm 0.17$ SDS, available in 11 subjects) by 0.51 SDS $(3.7 \mathrm{~cm} ; \mathrm{p}<0.02$; $95 \%$ CI $0.10-0.92$ SDS). Modified intent-totreat analysis in 62 patients treated for at least 6 months indicated a similar GH effect on last observed height SDS (0.52 SDS; $3.8 \mathrm{~cm} ; \mathrm{p}<0.001 ; 95 \%$ CI 0.22-0.82 SDS) and no important dropout bias.

Conclusion: GH treatment increases adult height in peripubertal children with marked idiopathic short stature.

\section{Growth hormone (GH) treatment to final height in children with idiopathic short stature: evidence for a dose effect}

Wit JM, Rekers-Mombarg LT, Cutler GB, Crowe B, Beck TJ, Roberts K, Gill A, Chaussain JL, Frisch H, Yturriaga R, Attanasio AF

Leiden University Medical Center, Leiden, the Netherlands

j.m.wit@lumc.nl

J Pediatr 2005; 146:45-53

Background: Whether GH treatment increases adult height in subjects with idiopathic short stature (ISS) is not known.

Methods: In an open-label study, patients with ISS (-3.2 SDS) were treated with GH at $0.24 \mathrm{mg} / \mathrm{kg} / \mathrm{week}$, $0.24 \mathrm{mg} / \mathrm{kg} /$ week for the first year and at $0.37 \mathrm{mg} / \mathrm{kg} /$ week thereafter $(0.24 \rightarrow 0.37)$, or $0.37 \mathrm{mg} / \mathrm{kg} /$ week. Results: Final height was evaluated in 50 patients at study completion (mean treatment duration 6.5 years). Mean height SDS increased by $1.55,1.52$, and 1.85 SDS, respectively, for the 3 dose groups. For the primary comparison between the 0.37 and $0.24 \mathrm{mg} / \mathrm{kg} /$ week dose groups, the mean treatment difference (adjusted for differences in baseline predicted height SDS) was 0.57 SDS $(3.6 \mathrm{~cm} ; \mathrm{p}=0.025)$. Final height measurements were within the normal adult height range for $94 \%$ of patients randomized to the $0.37 \mathrm{mg} / \mathrm{kg} /$ week dose who continued to final height.

Conclusion: GH treatment dose-dependently increases final height in children with idiopathic short stature.

FDA approval of GH therapy for ISS was an important event in the GH field this year. The randomized controlled study by Leschek et al. showed a minimal $(3.7 \mathrm{~cm})$ increase in adult height in peripubertal subjects with ISS. However, the age at initiation of treatment was late (between 12 and 13 years on average), and GH was given 3 times per week: optimal conditions were certainly not fulfilled to get a 
spectacular result. In the study by Wit et al., the height increase with GH treatment was dose-dependent. The subjects in this work were about 2 years younger than in the work by Leschek et al., and treatment duration was 2 years longer. These two papers illustrate the difficulty in performing longterm trials in the growth field, in particular with placebo-treated or untreated control groups. Notably, the spontaneous height gain of the placebo group in the study by Leschek et al. was $0.42 \pm 0.25$ SDS. Other controlled studies have shown that the spontaneous height gain in untreated subjects was 0.4 to 0.8 SDS $[13,14]$. Assuming a similar spontaneous height gain in the subjects with ISS from the study by Wit et al., the true increase in final height attributable to GH treatment may be less impressive, although still important (0.75-1.4 SDS). Overall, these studies showed encouraging results for treating children with ISS. However, optimization of GH treatment remains to be determined in this indication. Otherwise, ISS remains an unacceptable diagnosis. It is certainly a basket full of unrelated entities. For instance, SHOX mutations, which are responsible for Leri-Weill dyschondrosteosis, have been found in about $2 \%$ of apparently idiopathic short stature [15]. Our next goal should be to sort ISS into mechanistic categories.

\section{New genes \\ One phenotype, two genotypes}

\section{Heterozygous TGFBR2 mutations in Marfan syndrome}

Mizuguchi T, Collod-Beroud G, Akiyama T, Abifadel M, Harada N, Morisaki T, Allard D, Varret M, Claustres M, Morisaki H, Ihara M, Kinoshita A, Yoshiura K, Junien C, Kajii T, Jondeau G, Ohta T, Kishino T, Furukawa Y, Nakamura Y, Niikawa N, Boileau C, Matsumoto N

Department of Human Genetics, Nagasaki University Graduate School of Biomedical Sciences, Nagasaki, Japan Nat Genet 2004;36:855-860

Background: Marfan syndrome is a dominantly inherited disorder of fibrous connective tissue affecting the skeletal, cardiovascular and ocular systems, with an incidence of 1 in 10,000. Approximately $80 \%$ of Marfan cases are due to inactivating mutations in the gene encoding fibrillin (FBN1) at 15q21.1. A second type of the disorder has been associated with a second locus, MFS2, at 3p25-p24.2 in a large French family.

Methods: Identification of a 3p24.1 chromosomal breakpoint disrupting the gene encoding TGF $\beta$ receptor 2 (TGFBR2) in a Japanese individual with Marfan syndrome led the authors to consider TGFBR2 as the gene underlying association with Marfan syndrome at the MSF2 locus.

Results: The mutation 1524G $\rightarrow$ A in TGFBR2 (causing the amino acid substitution Q508Q) resulted in abnormal splicing and segregated with MFS2. Three other missense mutations were identified in 4 unrelated probands, which led to loss of function of TGF $\beta$-signaling activity on extracellular matrix formation.

Conclusion: These results show that heterozygous mutations in TGFBR2 are also associated with inherited connective-tissue disorders.

The TGFs are multifunctional growth factors that signal through a pathway of transmembrane serine-threonine kinase receptors and intracellular signaling molecules of the SMAD family. TGF $\beta$ regulates many cellular processes, including proliferation, cell cycle arrest, apoptosis, differentiation and extracellular matrix formation. All the missense mutations identified in this work occur at conservative amino-acid residues in the serine-threonine kinase domain of the receptor, inactivating the receptor. This study shows that perturbation of TGF $\beta$ signaling contributes to the pathogenesis of extracellular matrix disorders. TGFBR2 was previously reported to be a potent tumor suppressor gene and mutations described in TGFBR2 have been associated with colorectal cancer. Marfan syndrome, however, is not considered to be associated with a high risk of cancer. Why mutations in the same gene may lead to completely different diseases remains to be investigated. 


\section{Growth retardation and abnormal maternal behavior in mice lacking testicular orphan nuclear receptor 4}

Collins LL, Lee YF, Heinlein CA, Liu NC, Chen YT, Shyr CR, Meshul CK, Uno H, Platt KA, Chang C

George Whipple Laboratory for Cancer Research, Departments of Pathology, Urology, and Radiation Oncology, and Cancer Center, University of Rochester Medical Center, Rochester, N.Y., USA

Proc Natl Acad Sci USA 2004;101:15058-15063

Background: Testicular orphan nuclear receptor 4 (TR4) is a member of the nuclear receptor superfamily. It is closely related to the retinoid X receptor (RXR). However, its natural ligand is unknown. Methods: Generation of mice lacking TR4 by means of targeted gene disruption (TR4 $\left.{ }^{-l-}\right)$.

Results: The number of TR $4^{-/}$pups generated by the mating of TR $4^{+/-}$mice is well under that predicted by the normal Mendelian ratio, and TR $4^{-1-}$ mice demonstrate high rates of early postnatal mortality. Birth weight was normal, but significant growth retardation appeared from the first week of life. GH production was normal, but serum IGF-1 was low. Additionally, TR $4^{-1-}$ females show defects in reproduction and maternal behavior, with pups of TR $4^{-1-}$ dams dying soon after birth with no indication of milk intake.

Conclusion: TR4 plays important roles in growth, embryonic and early postnatal pup survival, female reproductive function, and maternal behavior.

Members of the nuclear receptor superfamily are known to play important roles in differentiation, development, homeostasis, and metabolism, as well as in disease development and progression. In rodents, TR4 expression can be detected in peripheral organs, such as the adrenal gland, spleen, testis, thyroid, pituitary, and prostate. A substantial difficulty in determining the function of TR4 has been the inability to identify ligands through which the receptors are activated. In response to this dilemma, creation of mammalian gene knockout models has become a successful strategy to study the physiological roles of orphan receptors in vivo. In this study, the authors described the phenotype associated with TR4 disruption: increased embryologic mortality, significant growth reduction (as early as postnatal day 2), reduction of IGF-1 levels without a corresponding reduction in GH (partial $\mathrm{GH}$ resistance?), reduction in the fertility of both males and females, lack of maternal bonding behavior (associated with reduced oxytocin expression in the hypothalamic regions). Recently, impaired cerebellar function has also been described in these mice [16]. Although the phenotype was complex, the effect of TR4 disruption on growth was one of the more prominent. When the endogenous ligand of TR4 will be discovered, new insights into growth pathways will certainly emerge. One wonders whether these receptors are orphans by nature, have an unknown ligand, or precede the ligand in terms of evolution.

\section{Food for thought}

Insights into GH signaling

\section{Short stature caused by a biologically inactive mutant growth hormone (GH-C53S)}

Besson A, Salemi S, Deladoey J, Vuissoz JM, Eble A, Fluck C, Mullis PE

University Children's Hospital, Pediatric Endocrinology and Metabolism, Inselspital Bern, Switzerland; Medizinische Klinik-Innenstadt, Munich University, Munich, Germany, and Department of Pharmacology, University of Bern, Bern, Switzerland

J Clin Endocrinol Metab 2005;90:2493-2499

Background: Human GH has two disulfide bridges. The importance of this bridge in mediating the $\mathrm{GH} / \mathrm{GHR}$ interaction in humans is unknown. 
Methods: A homozygous missense mutation (G705C) was identified in the GH1 gene of a Serbian patient with short stature (-3.6 SD at age 9). This leads to the absence of the disulfide bridge Cys-53 to Cys165. To study the impact of this mutation, GH-receptor (GHR) binding and Jak2/Stat5 activation experiments were performed.

Results: GHR binding and Jak2/Stat5 signaling pathway activation were significantly reduced in the mutant GH-C53S compared with wt-GH at physiological GH concentration. Higher concentrations $(400 \mathrm{ng} / \mathrm{ml})$ were required for this mutant to elicit responses similar to wt-GH.

Conclusion: The absence of the disulfide bridge Cys-53 to Cys-165 affects the binding affinity of GH for the GHR and subsequently the potency of GH to activate the Jak2/Stat 5 signaling pathway.

\section{Suppressor of cytokine signaling-2 deficiency induces molecular and metabolic changes that partially overlap with growth hormone-dependent effects}

Rico-Bautista E, Greenhalgh CJ, Tollet-Egnell P, Hilton DJ, Alexander WS, Norstedt G, Flores-Morales A Department of Molecular Medicine, Karolinska Institute, Stockholm, Sweden

Mol Endocrinol 2005; 19:781-793

Background: Suppressor of cytokine signaling-2 (SOCS2)-deficient (SOCS2 ${ }^{-1-}$ ) mice grow significantly larger than their littermates. The aim of this study was to identify genes and metabolic parameters that might contribute to the SOCS2 ${ }^{-1-}$ phenotype.

Methods: Metabolic studies in SOCS2 ${ }^{-1-}$ male mice. cDNA microarray analysis from liver tissue, and real-time quantitative RT PCR of 6 genes expressed in the liver.

Results: Although SOCS2 deficiency induces significant changes in hepatic gene expression, only a fraction of these overlap with known GH-induced effects in the liver. These animals overexpressed IGFbinding protein-3 mRNA in the liver and had increased levels of circulating IGF-binding protein-3. Other GH-like effects included diminished serum triglycerides and down-regulation of lipoprotein lipase in adipose tissue. SOCS2 $2^{-1-}$ mice and their wild-type littermates had comparable glucose or insulin tolerance tests, and insulin signaling was normal in primary hepatocytes from SOCS2 ${ }^{-1-}$ mice. The expression of peroxisome proliferator-activated receptor- $\gamma$ coactivator- $1 \alpha$ (PGC1 $\alpha)$ mRNA was increased in skeletal muscle: this might contribute to normal glucose tolerance despite the apparent overactivity of the GH signaling pathway.

Conclusion: SOCS2 deficiency partially mimics a state of increased GH activity, but also results in changes that cannot be related to known GH effects.

These 2 papers contribute to a better understanding of the GH-signaling pathway. Biologically inactive GH was first suggested as a cause of short stature by Kowarski et al [17]. Although several mutations in the GH1 gene which were considered to lead to bio-inactive GH have since been described $[18,19]$, no clear correlation between laboratory/clinical phenotype, patient genotype, and familial segregation has been shown. The patient reported here had short stature, normal GH and GHBP but low IGF-1 and IGFBP3 levels, and a normal IGF-1 response to an IGF-1 generation test. The height gain under rhGH treatment was in the range of that observed in $\mathrm{GH}$-deficient patients. The GHR sequence was normal. By abolishing a disulfide bridge, the homozygous GH1 gene mutation described here likely altered the spatial arrangement of the GH protein. This decreased the binding of the mutant $\mathrm{GH}$ to the GHR and the capacity to activate STAT5 at physiological concentration. Overall, this is one of the most convincing demonstrations of the bio-inactive GH concept. Notably, bio-inactive IGF-1 has also been reported this year in 1 subject with short stature and very high levels of IGF-1 [20, 21]. This patient is described in the chapter by L. Sävendahl in this book.

SOCS2 ${ }^{-1-}$ mice and GH overexpression models have phenotypic similarities regarding somatic growth. However, hepatic mRNA IGF-1 expression as well as circulating IGF-1 levels are normal in these mice. The paper by Rico-Bautista et al. shows that the excessive growth phenotype of $\mathrm{SOCS2}^{-1-}$ animals may be due to the activation of a small subset of hepatic GH-regulated genes, including the IGFBP3 gene. An alternative explanation for the overgrowth phenotype could be that increased GH receptor signaling in extrahepatic target tissues, such as bone and muscle, might be more important than the hepatic effects of SOCS2 deficiency. They also showed that these mice had normal glucose tolerance 
and insulin sensitivity, and increased muscular expression of PGC-1 $\alpha$, a positive regulator of glucose utilization, as opposed to the insulin resistance phenotype observed in GH overexpressing mice. The metabolic changes in relation to glucose and lipid metabolism suggest that the growth-promoting actions of GH can be dissociated from its diabetogenic effects. Therapies targeting the inhibition of SOCS2 could result in beneficial effects that mimic some of the GH anabolic, lipolytic, and growthpromoting activities while avoiding the diabetogenic actions associated with direct GH treatment.

\section{References}

1. Metcalf D, Greenhalgh CJ, Viney E, Willson TA, Starr R, Nicola NA, et al: Gigantism in mice lacking suppressor of cytokine signalling-2. Nature 2000;405:1069-1073.

2. Prawitt D, Enklaar T, Gartner-Rupprecht B, Spangenberg C, Oswald M, Lausch E, et al: Microdeletion of target sites for insulator protein CTCF in a chromosome $11 \mathrm{p} 15$ imprinting center in Beckwith-Wiedemann syndrome and Wilm's tumor. Proc Natl Acad Sci USA 2005;102:4085-4090.

3. Eggermann T, Meyer E, Obermann C, Heil I, Schuler H, Ranke MB, et al: Is maternal duplication of 11p15 associated with Silver-Russell syndrome? J Med Genet 2005;42:e26.

4. Lundgren EM, Cnattingius S, Jonsson B, Tuvemo T: Intellectual and psychological performance in males born small for gestational age with and without catch-up growth. Pediatr Res 2001;50:91-96.

5. Burman P, Broman JE, Hetta J, Wiklund I, Erfurth EM, Hagg E, et al: Quality of life in adults with growth hormone (GH) deficiency: response to treatment with recombinant human GH in a placebo-controlled 21-month trial. J Clin Endocrinol Metab 1995;80:3585-3590.

6. Francis DD, Szegda K, Campbell G, Martin WD, Insel TR: Epigenetic sources of behavioral differences in mice. Nat Neurosci 2003;6:445-446.

7. Gunnell D, Okasha M, Smith GD, Oliver SE, Sandhu J, Holly JM: Height, leg length, and cancer risk: a systematic review. Epidemiol Rev 2001;23:313-342.

8. Ajiki W, Tsukuma H, Oshima A, Research Group for Population-based Cancer Registration in Japan: Cancer incidence and incidence rates in Japan in 1999: estimates based on data from 11 population-based cancer registries. Jpn J Clin Oncol 2004;34:352-356.

9. Stephure DK: Impact of growth hormone supplementation on adult height in turner syndrome: results of the Canadian randomized controlled trial. J Clin Endocrinol Metab 2005;90:3360-3366.

10. Cave CB, Bryant J, Milne R: Recombinant growth hormone in children and adolescents with Turner syndrome. Cochrane Database Syst Rev 2003(3):CD003887.

11. Eiholzer U: Deaths in children with Prader-Willi syndrome. A contribution to the debate about the safety of growth hormone treatment in children with PWS. Horm Res 2005;63:33-39.

12. Nagai T, Obata K, Tonoki H, Temma S, Murakami N, Katada Y, et al: Cause of sudden, unexpected death of PraderWilli syndrome patients with or without growth hormone treatment. Am J Med Genet A 2005;136:45-48.

13. Buchlis JG, Irizarry L, Crotzer BC, Shine BJ, Allen L, MacGillivray MH: Comparison of final heights of growth hormonetreated vs. untreated children with idiopathic growth failure. J Clin Endocrinol Metab 1998;83:1075-1079.

14. Coutant R, Rouleau S, Despert F, Magontier N, Loisel D, Limal JM: Growth and adult height in GH-treated children with nonacquired GH deficiency and idiopathic short stature: the influence of pituitary magnetic resonance imaging findings. J Clin Endocrinol Metab 2001;86:4649-4654.

15. Schneider KU, Sabherwal N, Jantz K, Roth R, Muncke N, Blum WF, et al: Identification of a major recombination hotspot in patients with short stature and SHOX deficiency. Am J Hum Genet 2005;77:89-96.

16. Chen YT, Collins LL, Uno H, Chang C: Deficits in motor coordination with aberrant cerebellar development in mice lacking testicular orphan nuclear receptor 4. Mol Cell Biol 2005;25:2722-2732.

17. Kowarski AA, Schneider J, Ben-Galim E, Weldon VV, Daughaday WH: Growth failure with normal serum RIA-GH and low somatomedin activity: somatomedin restoration and growth acceleration after exogenous GH. J Clin Endocrinol Metab 1978;47:461-464.

18. Takahashi Y, Shirono H, Arisaka O, Takahashi K, Yagi T, Koga J, et al: Biologically inactive growth hormone caused by an amino acid substitution. J Clin Invest 1997;100:1159-1165.

19. Millar DS, Lewis MD, Horan M, Newsway V, Easter TE, Gregory JW, et al: Novel mutations of the growth hormone 1 (GH1) gene disclosed by modulation of the clinical selection criteria for individuals with short stature. Hum Mutat 2003;21:424-440.

20. Walenkamp MJ, Karperien M, Pereira AM, Hilhorst-Hofstee Y, van Doorn J, Chen JW, et al: Homozygous and heterozygous expression of a novel insulin-like growth factor-I mutation. J Clin Endocrinol Metab 2005;90:2855-2864.

21. Denley A, Wang CC, McNeil KA, Walenkamp MJ, van Duyvenvoorde H, Wit JM, et al: Structural and functional characteristics of the Val44Met insulin-like growth factor I missense mutation: correlation with effects on growth and development. Mol Endocrinol 2005;19:711-721. 